\title{
The (adaptive) benefits of sex
}

DOI:

10.1038/nrmicro1755
What is the evolutionary benefit of sex? The answer has remained elusive for more than a century. The Fisher-Muller model proposes that sex, through recombination, eliminates competition among beneficial mutations that have arisen in different genetic backgrounds. Instead, recombination brings these beneficial mutations together and speeds up adaptation. This model has extensive theoretical support, but has been difficult to test experimentally. Reporting in PLoS Biology, Tim Cooper now provides direct experimental support for the Fisher-Muller model.

Ideally, experimental testing of the Fisher-Muller model would involve comparing the effects of recombination on the rate of adaptation in treatment regimes that differ only in the extent of competition between beneficial mutations, and would exclude the effects of all other environmental factors. Bacterial sex provides a simple model, and the authors of a previous study identified conditions under which the absence of a DNA-repair gene controlled the rate of adaptation in evolving populations of Escherichia coli by increasing the rate at which new mutations were produced by approximately 30 -fold. Here, Cooper used the same strains and environmental conditions to set up a simple microbial system, in which high and low mutation-rate treatments differed only in the supply of new mutations, and therefore the degree of competition between beneficial mutations. Recombination was mediated by introducing the F plasmid into recombination proficient $\left(\mathrm{rec}^{+}\right)$ancestral strains of E. coli. The F plasmid integrates into the bacterial chromosome, where it facilitates gene transfer into a recipient cell by conjugation. Control recombination-deficient $\left(\mathrm{rec}^{-}\right)$strains were made by deleting an F-plasmid gene that is essential for plasmid conjugation. Eight replicate lines were evolved under each of four different conditions ( $\mathrm{rec}^{+}$and $\mathrm{rec}^{-}$strains with both high and low mutation rates) and the effects on adaptation were measured by comparing differences in the fitness increases that were achieved by evolved populations.

Under the high mutation-rate treatment, in which beneficial mutations competed for fixation, recombination provided an advantage, increasing the rate of adaptation by approximately three-fold compared with non-recombining strains. The Fisher-Muller model also predicts that recombination reduces competition between beneficial mutations. To test this, Cooper sequenced candidate loci and found a beneficial

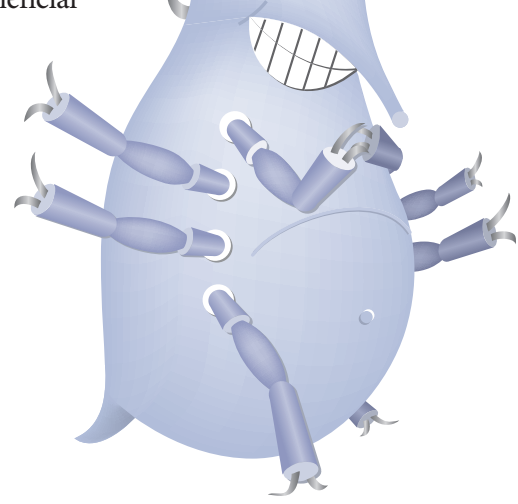

mutation in six of the high mutationrate lines. He then compared the dynamics of this beneficial mutation in the $\mathrm{rec}^{+}$and $\mathrm{rec}^{-}$populations. More generations were required for the mutation to spread in the absence of recombination, and overall the mutation conferred a lower competitive advantage, indicating that there was interference between the competing beneficial mutations.

This study proves that recombination increases the rate of adaptation, but only when there are competing beneficial mutations in the population. These findings provide much-needed evidence to support the Fisher-Muller model for the evolution of sex.

Sharon Ahmad

ORIGINAL RESEARCH PAPER Cooper, T. F. Recombination speeds adaptation by reducing competition between beneficial mutations in populations of Escherichia coli. PLoS Biol. 5, e225 (2007)

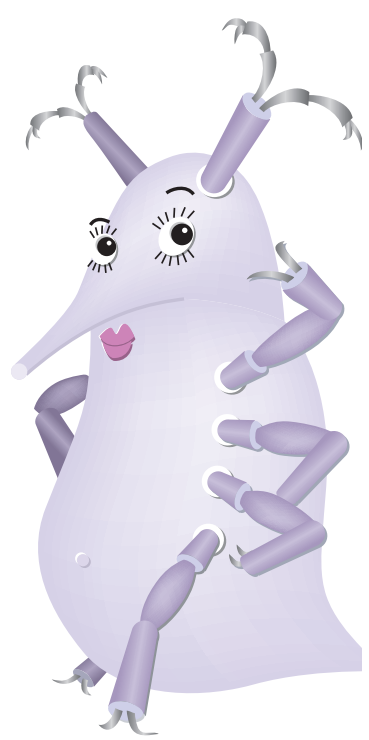

